\title{
FINITE-TIME STABILITY OF CONTINUOUS AUTONOMOUS SYSTEMS*
}

\author{
SANJAY P. BHAT ${ }^{\dagger}$ AND DENNIS S. BERNSTEIN $\ddagger$
}

\begin{abstract}
Finite-time stability is defined for equilibria of continuous but non-Lipschitzian autonomous systems. Continuity, Lipschitz continuity, and Hölder continuity of the settling-time function are studied and illustrated with several examples. Lyapunov and converse Lyapunov results involving scalar differential inequalities are given for finite-time stability. It is shown that the regularity properties of the Lyapunov function and those of the settling-time function are related. Consequently, converse Lyapunov results can only assure the existence of continuous Lyapunov functions. Finally, the sensitivity of finite-time-stable systems to perturbations is investigated.
\end{abstract}

Key words. stability, finite-time stability, non-Lipschitzian dynamics

AMS subject classifications. 34D99, 93D99

PII. S0363012997321358

1. Introduction. The object of this paper is to provide a rigorous foundation for the theory of finite-time stability of continuous autonomous systems and motivate a closer examination of finite-time stability as a possible objective in control design.

Classical optimal control theory provides several examples of systems that exhibit convergence to the equilibrium in finite time [17]. A well-known example is the double integrator with bang-bang time-optimal feedback control [2]. These examples typically involve dynamics that are discontinuous. Discontinuous dynamics, besides making a rigorous analysis difficult (see [9]), may also lead to chattering [10] or excite high frequency dynamics in applications involving flexible structures. Reference [8] considers finite-time stabilization using time-varying feedback controllers. However, it is well known that the stability analysis of time-varying systems is more complicated than that of autonomous systems. Therefore, with simplicity as well as applications in mind, we focus on continuous autonomous systems.

Finite-settling-time behavior of systems with continuous dynamics is considered in [3], [4], [11], [19], [21]. However, a detailed analysis of such systems has not been carried out. In particular, a precise formulation of finite-time stability is lacking, while little is known about the settling-time function. Furthermore, while references [3], [4], [11], [19] present Lyapunov conditions for finite-time stability, neither rigorous proofs nor converse results can be found. Reference [21] suggests, based on a scalar example, that systems with finite-settling-time dynamics possess better disturbance rejection and robustness properties. However, no precise results exist for multidimensional systems. This paper attempts to fill these gaps.

In section 2, we define finite-time stability for equilibria of continuous autonomous systems that have unique solutions in forward time. Continuity and forward uniqueness render the solutions continuous functions of the initial conditions, so that the

${ }^{*}$ Received by the editors May 12, 1997; accepted for publication (in revised form) March 30, 1999; published electronically February 29, 2000.

http://www.siam.org/journals/sicon/38-3/32135.html

${ }^{\dagger}$ Department of Aerospace Engineering, Indian Institute of Technology, Powai, Mumbai 400076, India (bhat@aero.iitb.ernet.in).

${ }^{\ddagger}$ Department of Aerospace Engineering, The University of Michigan, Ann Arbor, MI 48109-2140 (dsbaero@engin.umich.edu). The research of this author was supported in part by Air Force Office of Scientific Research grant F49620-95-1-0019. 
solutions define a continuous semiflow on the state space. Uniqueness also makes it possible to define the settling-time function. Certain useful properties of the settling time function are established. It is shown by example that it is possible for the settling time to be unbounded in every neighborhood of the origin even if all solutions converge to the origin in finite time. A different example shows that the settling-time function may be continuous without being Hölder continuous at the origin.

In section 3 we define finite-time repellers (called terminal repellers in [7], [23]), which are a special class of unstable equilibria that arise only in non-Lipschitzian systems. We show that a system having a finite-time repeller possesses multiple solutions starting at the finite-time repeller.

In section 4, we give a Lyapunov theorem for finite-time stability. Dini derivatives are used since Lyapunov functions are assumed to be only continuous. A converse result is shown to hold under the assumption that the settling-time function is continuous. In general, the converse result cannot be strengthened in its conclusion regarding the regularity of the Lyapunov function; that is, a system with a finite-time-stable equilibrium may not admit a Hölder continuous Lyapunov function. This is because Hölder continuity of the Lyapunov function necessarily implies Hölder continuity of the settling-time function at the origin. On the other hand, as mentioned above, there exist finite-time-stable systems with settling-time functions that are not Hölder continuous at the origin.

The existence of a Hölder continuous Lyapunov function assumes importance in section 5 where we investigate the sensitivity of stability properties to perturbations of systems with a finite-time-stable equilibrium under the assumption of the existence of a Lipschitz continuous Lyapunov function. Both persistent and vanishing perturbations are considered. It is shown that under certain conditions, finite-time-stable systems may exhibit better rejection of bounded persistent disturbances than Lipschitzian exponentially stable systems. It is also shown that finite-time stability is preserved under perturbations that are Lipschitz in the state.

2. Finite-time stability. Let $\|\cdot\|$ denote a norm on $\mathbb{R}^{n}$. The notions of openness, convergence, continuity, and compactness that we use refer to the topology generated on $\mathbb{R}^{n}$ by the norm $\|\cdot\|$. We use $\overline{\mathbb{R}}, \mathbb{R}_{+}$, and $\overline{\mathbb{R}}_{+}$to denote the extended, nonnegative, and extended nonnegative, real numbers, respectively. We also use $\overline{\mathcal{A}}$ and bd $\mathcal{A}$ to denote the closure and the boundary of the set $\mathcal{A}$, respectively. We will call a set $\mathcal{A} \subset \mathbb{R}^{n}$ bounded if $\overline{\mathcal{A}}$ is compact. Finally, we denote the composition of two functions $U: \mathcal{A} \rightarrow \mathcal{B}$ and $V: \mathcal{B} \rightarrow \mathcal{C}$ by $V \circ U: \mathcal{A} \rightarrow \mathcal{C}$.

Consider the system of differential equations

$$
\dot{y}(t)=f(y(t)),
$$

where $f: \mathcal{D} \rightarrow \mathbb{R}^{n}$ is continuous on an open neighborhood $\mathcal{D} \subseteq \mathbb{R}^{n}$ of the origin and $f(0)=0$. A continuously differentiable function $y: I \rightarrow \mathcal{D}$ is said to be a solution of (2.1) on the interval $I \subset \mathbb{R}$ if $y$ satisfies (2.1) for all $t \in I$. The continuity of $f$ implies that, for every $x \in \mathcal{D}$, there exist $\tau_{0}<0<\tau_{1}$ and a solution $y(\cdot)$ of (2.1) defined on $\left(\tau_{0}, \tau_{1}\right)$ such that $y(0)=x$ [12, Thm. I.1.1]. A solution $y$ is said to be right maximally defined if $y$ cannot be extended on the right (either uniquely or nonuniquely) to a solution of (2.1). Every solution of (2.1) has an extension that is right maximally defined [12, Thm. I.2.1]. For later use, we state the following result on bounded solutions of (2.1). For a proof, see [12, pp. 17-18] or [22, Thm. 3.3, p. 12].

Proposition 2.1. If $y:[0, \tau) \rightarrow \mathcal{D}$ is a right maximally defined solution of (2.1) such that $y(t) \in \mathcal{K}$ for all $t \in[0, \tau)$, where $\overline{\mathcal{K}} \subset \mathcal{D}$ is compact, then $\tau=\infty$. 
We will assume that (2.1) possesses unique solutions in forward time for all initial conditions except possibly the origin in the following sense: for every $x \in \mathcal{D} \backslash\{0\}$ there exists $\tau_{x}>0$ such that, if $y_{1}:\left[0, \tau_{1}\right) \rightarrow \mathcal{D}$ and $y_{2}:\left[0, \tau_{2}\right) \rightarrow \mathcal{D}$ are two right maximally defined solutions of $(2.1)$ with $y_{1}(0)=y_{2}(0)=x$, then $\tau_{x} \leq \min \left\{\tau_{1}, \tau_{2}\right\}$ and $y_{1}(t)=y_{2}(t)$ for all $t \in\left[0, \tau_{x}\right)$. Without any loss of generality, we may assume that for each $x, \tau_{x}$ is chosen to be the largest such number in $\overline{\mathbb{R}}_{+}$. In this case, we denote by $\psi(\cdot, x)$ or, alternatively, $\psi^{x}(\cdot)$ the unique solution of $(2.1)$ on $\left[0, \tau_{x}\right)$ satisfying $\psi(0, x)=x$. Note that $\psi^{x}$ cannot be extended on the right uniquely to a solution of (2.1) because if $\tau_{x}<\infty$, then as $t \rightarrow \tau_{x}$, either $\psi(t, x)$ approaches bd $\mathcal{D}$ [12, Thm. I.2.1], in which case $\psi^{x}$ cannot be extended on the right to a solution of (2.1), or $\psi(t, x)$ approaches 0 with (2.1) having nonunique solutions starting at 0 , in which case $\psi^{x}$ can be extended on the right to a solution of (2.1) in more than one way. If (2.1) has nonunique solutions in forward time for the initial condition 0 , then $\psi$ is defined on a relatively open subset of $\mathbb{R}_{+} \times \mathcal{D} \backslash\{0\}$ onto $\mathcal{D} \backslash\{0\}$. If (2.1) possesses a unique solution in forward time for the initial condition 0 , then $\psi$ is defined on a relatively open subset of $\mathbb{R}_{+} \times \mathcal{D}$ onto $\mathcal{D}$ and for each $x \in \mathcal{D}, \psi^{x}:\left[0, \tau_{x}\right) \rightarrow \mathcal{D}$ is the unique right maximally defined solution of (2.1) for the initial condition $x$. Uniqueness in forward time and the continuity of $f$ imply that $\psi$ is continuous on its domain of definition [12, Thm. I.3.4] and defines a local semiflow [6], [20, Ch. 2] on $\mathcal{D} \backslash\{0\}$ or $\mathcal{D}$, as the case may be. Various sufficient conditions for forward uniqueness in the absence of Lipschitz continuity can be found in [1], [9, sect. 10], [14], [22, sect. 1].

DeFinition 2.2. The origin is said to be a finite-time-stable equilibrium of (2.1) if there exists an open neighborhood $\mathcal{N} \subseteq \mathcal{D}$ of the origin and a function $T: \mathcal{N} \backslash\{0\} \rightarrow$ $(0, \infty)$, called the settling-time function, such that the following statements hold:

(i) Finite-time convergence: For every $x \in \mathcal{N} \backslash\{0\}, \psi^{x}$ is defined on $[0, T(x))$, $\psi^{x}(t) \in \mathcal{N} \backslash\{0\}$ for all $t \in[0, T(x))$, and $\lim _{t \rightarrow T(x)} \psi^{x}(t)=0$.

(ii) Lyapunov stability: For every open neighborhood $\mathcal{U}_{\varepsilon}$ of 0 there exists an open subset $\mathcal{U}_{\delta}$ of $\mathcal{N}$ containing 0 such that, for every $x \in \mathcal{U}_{\delta} \backslash\{0\}, \psi^{x}(t) \in \mathcal{U}_{\varepsilon}$ for all $t \in[0, T(x))$.

The origin is said to be a globally finite-time-stable equilibrium if it is a finite-timestable equilibrium with $\mathcal{D}=\mathcal{N}=\mathbb{R}^{n}$.

The following proposition shows that if the origin is a finite-time-stable equilibrium of (2.1), then (2.1) has a unique solution on $\mathbb{R}_{+}$for every initial condition in an open neighborhood of 0 , including 0 itself.

Proposition 2.3. Suppose the origin is a finite-time-stable equilibrium of (2.1). Let $\mathcal{N} \subseteq \mathcal{D}$ and let $T: \mathcal{N} \backslash\{0\} \rightarrow(0, \infty)$ be as in Definition 2.2. Then, $\psi$ is defined on $\mathbb{R}_{+} \times \mathcal{N}$ and $\psi(t, x)=0$ for all $t \geq T(x), x \in \mathcal{N}$, where $T(0) \triangleq 0$.

Proof. It can be shown that Lyapunov stability of the origin implies that $y \equiv 0$ is the unique solution $y$ of $(2.1)$ satisfying $y(0)=0$. This proves that $\mathbb{R}_{+} \times\{0\}$ is contained in the domain of definition of $\psi$ and $\psi^{0} \equiv 0$.

Now, let $\mathcal{N} \subseteq \mathcal{D}$ and $T$ be as in Definition 2.2 and let $x \in \mathcal{N} \backslash\{0\}$. Define

$$
\begin{aligned}
y(t) & =\psi(t, x), & & 0 \leq t<T(x), \\
& =0, & & T(x) \leq t .
\end{aligned}
$$

By construction, $y$ is continuously differentiable on $\mathbb{R}_{+} \backslash\{T(x)\}$ and satisfies (2.1) on $\mathbb{R}_{+} \backslash\{T(x)\}$. Also, it follows from the continuity of $f$ that

$$
\lim _{t \rightarrow T(x)^{-}} \dot{y}(t)=\lim _{t \rightarrow T(x)^{-}} f(y(t))=0=\lim _{t \rightarrow T(x)^{+}} \dot{y}(t),
$$


so that $y$ is continuously differentiable at $T(x)$ and satisfies (2.1). Thus $y$ is a solution of (2.1) on $\mathbb{R}_{+}$. To prove uniqueness, suppose $z$ is a solution of $(2.1)$ on $\mathbb{R}_{+}$satisfying $z(0)=x$. Then by the uniqueness assumption, $y$ and $z$ agree on $[0, T(x))$. By continuity, $y$ and $z$ must also agree on $[0, T(x)]$ so that $z(T(x))=0$. Lyapunov stability now implies that $z(t)=0$ for $t>T(x)$. This proves uniqueness. By the definition of $\psi$, it follows that $\psi^{x} \equiv y$. Thus $\psi^{x}$ is defined on $\mathbb{R}_{+}$and satisfies $\psi^{x}(t)=0$ on $[T(x), \infty)$ for every $x \in \mathcal{N}$. This proves the result.

Proposition 2.3 implies that if the origin is a finite-time-stable equilibrium of (2.1), then the solutions of (2.1) define a continuous global semiflow [20] on $\mathcal{N}$; that is, $\psi: \mathbb{R}_{+} \times \mathcal{N} \rightarrow \mathcal{N}$ is a (jointly) continuous function satisfying

$$
\begin{aligned}
\psi(0, x) & =x, \\
\psi(t, \psi(h, x)) & =\psi(t+h, x)
\end{aligned}
$$

for every $x \in \mathcal{N}$ and $t, h \in \mathbb{R}_{+}$. In addition, $\psi$ satisfies

$$
\psi(T(x)+t, x)=0
$$

for all $x \in \mathcal{N}$ and $t \in \mathbb{R}_{+}$.

Proposition 2.3 also indicates that it is reasonable to extend $T$ to all of $\mathcal{N}$ by defining $T(0)=0$. With a slight abuse of terminology, we will also call this extension the settling-time function. It is easy to see from Definition 2.2 that, for all $x \in \mathcal{N}$,

$$
T(x)=\inf \left\{t \in \mathbb{R}_{+}: \psi(t, x)=0\right\} .
$$

To illustrate finite-time stability, as well as for later use, we consider a scalar system with a finite-time-stable equilibrium.

Example 2.1. The right-hand side of the scalar system

$$
\dot{y}(t)=-k \operatorname{sign}(y(t))|y(t)|^{\alpha},
$$

where $\operatorname{sign}(0)=0, k>0$, and $\alpha \in(0,1)$, is continuous everywhere and locally Lipschitz everywhere except the origin. Hence every initial condition in $\mathbb{R} \backslash\{0\}$ has a unique solution in forward time on a sufficiently small time interval. The global semiflow for (2.7) is easily obtained by direct integration as

$$
\begin{aligned}
& \begin{aligned}
\mu(t, x) & =\operatorname{sign}(x)\left[|x|^{1-\alpha}-k(1-\alpha) t\right]^{\frac{1}{1-\alpha}}, & t<\frac{1}{k(1-\alpha)}|x|^{1-\alpha}, & x \neq 0, \\
& =0, & t \geq \frac{1}{k(1-\alpha)}|x|^{1-\alpha}, & x \neq 0,
\end{aligned} \\
& =0, \quad t \geq 0, \quad x=0 .
\end{aligned}
$$

It is clear from (2.8) that (i) in Definition 2.2 is satisfied with $\mathcal{D}=\mathcal{N}=\mathbb{R}$ and the settling-time function $T: \mathbb{R} \rightarrow \mathbb{R}_{+}$given by

$$
T(x)=\frac{1}{k(1-\alpha)}|x|^{1-\alpha} .
$$

Lyapunov stability follows by considering, for instance, the Lyapunov function $V(x)=$ $x^{2}$. Thus the origin is a globally finite-time-stable equilibrium for (2.7). Note that $T$ is Hölder continuous but not Lipschitz continuous at the origin.

The following proposition investigates the properties of the settling-time function of a finite-time-stable system.

Proposition 2.4. Suppose the origin is a finite-time-stable equilibrium of (2.1). Let $\mathcal{N} \subseteq \mathcal{D}$ be as in Definition 2.2 and let $T: \mathcal{N} \rightarrow \mathbb{R}_{+}$be the settling-time function. Then the following statements hold. 
(i) If $x \in \mathcal{N}$ and $t \in \mathbb{R}_{+}$, then

$$
T(\psi(t, x))=\max \{T(x)-t, 0\} .
$$

(ii) $T$ is continuous on $\mathcal{N}$ if and only if $T$ is continuous at 0.

(iii) For every $r>0$, there exists an open neighborhood $\mathcal{U}_{r} \subset \mathcal{N}$ of 0 such that, for every $x \in \mathcal{U}_{r} \backslash\{0\}$,

$$
T(x)>r\|x\| .
$$

Proof. (i) The result follows from (2.6), (2.4), and (2.5). 0 .

(ii) Necessity is immediate. To prove sufficiency, suppose that $T$ is continuous at Let $z \in \mathcal{N}$ and consider a sequence $\left\{z_{m}\right\}$ in $\mathcal{N}$ that converges to $z$. Let $\tau^{-}=$ $\liminf _{m \rightarrow \infty} T\left(z_{m}\right)$ and $\tau^{+}=\limsup _{m \rightarrow \infty} T\left(z_{m}\right)$. Note that both $\tau^{-}$and $\tau^{+}$are in $\overline{\mathbb{R}}_{+}$and

$$
\tau^{-} \leq \tau^{+}
$$

Next, let $\left\{z_{l}^{+}\right\}$be a subsequence of $\left\{z_{m}\right\}$ such that $T\left(z_{l}^{+}\right) \rightarrow \tau^{+}$as $l \rightarrow \infty$. The sequence $\left\{\left(T(z), z_{l}^{+}\right)\right\}$converges in $\mathbb{R}_{+} \times \mathcal{N}$ to $(T(z), z)$. By continuity and equation (2.5), $\psi\left(T(z), z_{l}^{+}\right) \rightarrow \psi(T(z), z)=0$ as $l \rightarrow \infty$. Since $T$ is assumed to be continuous at $0, T\left(\psi\left(T(z), z_{l}^{+}\right)\right) \rightarrow T(0)=0$ as $l \rightarrow \infty$. Using (2.10) with $t=T(z)$ and $x=z_{l}^{+}$, we obtain $\max \left\{T\left(z_{l}^{+}\right)-T(z), 0\right\} \rightarrow 0$ as $l \rightarrow \infty$. Thus $\max \left\{\tau^{+}-T(z), 0\right\}=0$, that is,

$$
\tau^{+} \leq T(z)
$$

Now, let $\left\{z_{l}^{-}\right\}$be a subsequence of $\left\{z_{m}\right\}$ such that $T\left(z_{l}^{-}\right) \rightarrow \tau^{-}$as $l \rightarrow \infty$. It follows from (2.12) and (2.13) that $\tau^{-} \in \mathbb{R}_{+}$. Therefore, the sequence $\left\{\left(T\left(z_{l}^{-}\right), z_{l}^{-}\right)\right\}$ converges in $\mathbb{R}_{+} \times \mathcal{N}$ to $\left(\tau^{-}, z\right)$. Since $\psi$ is continuous, it follows that $\psi\left(T\left(z_{l}^{-}\right), z_{l}^{-}\right) \rightarrow$ $\psi\left(\tau^{-}, z\right)$ as $l \rightarrow \infty$. Equation (2.5) implies that $\psi\left(T\left(z_{l}^{-}\right), z_{l}^{-}\right)=0$ for each $l$. Hence $\psi\left(\tau^{-}, z\right)=0$ and, by $(2.6)$,

$$
T(z) \leq \tau^{-} .
$$

From (2.12), (2.13), and (2.14) we conclude that $\tau^{-}=\tau^{+}=T(z)$ and hence $T\left(z_{m}\right) \rightarrow$ $T(z)$ as $m \rightarrow \infty$.

(iii) Let $r>0$. The function $\|f(\cdot)\|$ is continuous on $\mathcal{D}$ and $f(0)=0$ so that the set $\Omega_{r}=\left\{x \in \mathcal{N}:\|f(x)\|<\frac{1}{r}\right\}$ is open and contains 0. By Lyapunov stability, there exists an open set $\mathcal{U}_{r}$ such that $0 \in \mathcal{U}_{r} \subset \mathcal{N}$ and $\psi(t, x) \in \Omega_{r}$ for every $t \in \mathbb{R}_{+}$and $x \in \mathcal{U}_{r}$. Letting $x \in \mathcal{U}_{r} \backslash\{0\}$, we have

$$
0=\psi(T(x), x)=x+\int_{0}^{T(x)} f(\psi(t, x)) d t
$$

so that

$$
\|x\|=\left\|-\int_{0}^{T(x)} f(\psi(t, x)) d t\right\| \leq \int_{0}^{T(x)}\|f(\psi(t, x))\| d t<\frac{T(x)}{r},
$$

which proves the result. 


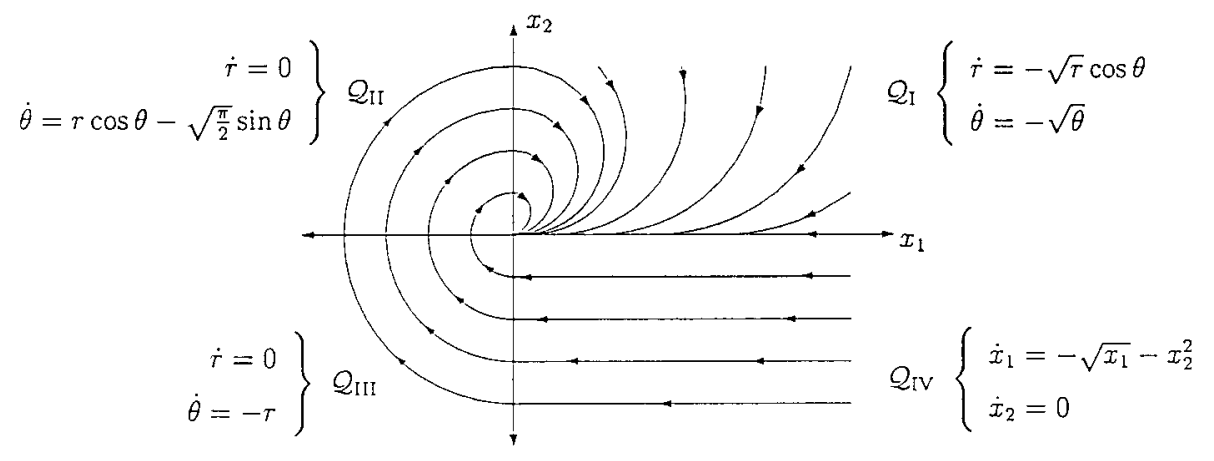

FIG. 2.1. Finite-time stability with discontinuous settling-time function.

Proposition 2.4 (ii) is significant because, in general, finite-time stability does not imply that the settling-time function $T$ is continuous at the origin. Indeed, as the following example shows, the settling-time function can be unbounded in every neighborhood of the origin.

Example 2.2. Consider the system (2.1) where the vector field $f: \mathbb{R}^{2} \rightarrow \mathbb{R}^{2}$ is defined on the quadrants

$$
\begin{aligned}
& \mathcal{Q}_{\mathrm{I}}=\left\{x \in \mathbb{R}^{2} \backslash\{0\}: x_{1} \geq 0, x_{2} \geq 0\right\}, \quad \mathcal{Q}_{\mathrm{II}}=\left\{x \in \mathbb{R}^{2}: x_{1}<0, x_{2} \geq 0\right\}, \\
& \mathcal{Q}_{\mathrm{III}}=\left\{x \in \mathbb{R}^{2}: x_{1} \leq 0, x_{2}<0\right\}, \quad \mathcal{Q}_{\mathrm{IV}}=\left\{x \in \mathbb{R}^{2}: x_{1}>0, x_{2}<0\right\},
\end{aligned}
$$

as shown in Figure 2.1, with $f(0)=0, r>0, \theta \in[0,2 \pi)$, and $x=\left(x_{1}, x_{2}\right)=$ $(r \cos \theta, r \sin \theta)$. It is easy to show that the vector field $f$ is continuous on $\mathbb{R}^{2}$ and locally Lipschitz everywhere on $\mathbb{R}^{2}$ except on the positive $x_{1}$-axis, denoted by $\mathcal{X}_{1}^{+}$, the negative $x_{2}$-axis, denoted by $\mathcal{X}_{2}^{-}$, and the origin. Since the derivative of $x_{2}^{2}$ along the solutions of (2.1) is nonpositive in a sufficiently small neighborhood of every point $x \in \mathcal{X}_{1}^{+}$, every solution $y(\cdot)$ of $(2.1)$ that satisfies $y(0) \in \mathcal{X}_{1}^{+}$satisfies $y(t) \in \mathcal{X}_{1}^{+}$for $t>0$ sufficiently small, while on $\mathcal{X}_{1}^{+}, f$ is simply given by $\dot{x}_{1}=-\sqrt{x_{1}}, \dot{x}_{2}=0$ which is easily seen to have unique solutions for initial conditions in $\mathcal{X}_{1}^{+}$. In fact, by Example 2.1 , solutions starting in $\mathcal{X}_{1}^{+}$converge to the origin in finite time. The vector field $f$ is also transversal to $\mathcal{X}_{2}^{-}$at every point in $\mathcal{X}_{2}^{-}$. Hence it follows from [14, Prop. $2.2]$ or $\left[9\right.$, Lem. 2, p. 107] that initial conditions in $\mathcal{X}_{2}^{-}$possess unique solutions in forward time. Thus (2.1) possesses a unique solution in forward time for every initial condition in $\mathbb{R}^{2} \backslash\{(0,0)\}$.

We show that the system given in Figure 2.1 has a globally finite-time-stable equilibrium at the origin and demonstrate a sequence $\left\{x_{m}\right\}$ in $\mathbb{R}^{2}$ such that $x_{m} \rightarrow 0$ and $T\left(x_{m}\right) \rightarrow \infty$, where $T$ is the settling-time function.

Lyapunov stability of the origin is easily verified using the Lyapunov function $x_{1}^{2}+x_{2}^{2}$. To show global finite-time convergence, we show that solutions starting in $\mathcal{Q}_{\text {IV }}$ and $\mathcal{Q}_{\text {III }} \cup \mathcal{Q}_{\text {II }}$ enter $\mathcal{Q}_{\text {III }}$ and $\mathcal{Q}_{\text {I }}$, respectively, in a finite amount of time, while solutions starting in $\mathcal{Q}_{\mathrm{I}}$ converge to the origin in finite time.

On $\mathcal{Q}_{\mathrm{IV}}, \dot{x_{2}}=0$ and $\dot{x_{1}} \leq-x_{2}^{2}<0$ so that after a finite amount of time (that depends on the initial condition) every solution starting in $\mathcal{Q}_{\mathrm{IV}}$ enters $\mathcal{Q}_{\mathrm{III}}$. Since $r \cos \theta-\sqrt{\frac{\pi}{2}} \sin \theta \leq \max \left\{-\sqrt{\frac{\pi}{2}},-r\right\}$ for $r>0$ and $\theta \in\left[\frac{\pi}{2}, \pi\right]$, it follows that $\dot{r}=0$ and $\dot{\theta} \leq \max \left\{-\sqrt{\frac{\pi}{2}},-r\right\}<0$ on $\mathcal{Q}_{\mathrm{III}} \cup \mathcal{Q}_{\mathrm{II}}$ so that every solution starting in $\mathcal{Q}_{\mathrm{III}} \cup \mathcal{Q}_{\mathrm{II}}$ enters $\mathcal{Q}_{\mathrm{I}}$ after a finite amount of time. Now, $\mathcal{Q}_{\mathrm{I}}$ is positively invariant. Hence, if 
a solution $y$ starting in $\mathcal{Q}_{\text {I }}$ does not converge to the origin for a sufficiently long time, then, since the scalar equation $\dot{\theta}=-\sqrt{\theta}$ has the origin as a finite-time-stable equilibrium by Example 2.1, $y$ converges to $\mathcal{X}_{1}^{+}$in finite time. We have already seen that solutions in $\mathcal{X}_{1}^{+}$converge to the origin in finite-time. Thus the origin is a globally finite-time-stable equilibrium.

Now consider the sequence $\left\{x_{m}\right\}$, where $x_{m}=\left(x_{m 1}, x_{m 2}\right)=\left(0,-\frac{1}{m}\right), m=$ $1,2, \ldots$, in $\mathcal{X}_{2}^{-}$. Thus $\left\{x_{m}\right\}$ lies in $\mathcal{X}_{2}^{-}$and $x_{m} \rightarrow 0$ as $m \rightarrow \infty$. Since $\dot{\theta}=-r$ on $\mathcal{Q}_{\text {III }}$, for every $m$, the time taken by the solution $y_{m}$ starting at $x_{m}$ to enter $\mathcal{Q}_{\text {II }}$ is equal to $\frac{\pi}{2 \sqrt{x_{m 1}^{2}+x_{m 2}^{2}}}=\frac{m \pi}{2}$. Since $y_{m}$ must enter $\mathcal{Q}_{\mathrm{II}}$ before converging to the origin, it follows that $T\left(x_{m}\right) \geq \frac{m \pi}{2}$ for every $m$ and hence $T\left(x_{m}\right) \rightarrow \infty$.

Proposition 2.4 (iii), which is equivalent to the statement that $\frac{\|x\|}{T(x)} \rightarrow 0$ as $x \rightarrow$ 0 , implies that the settling-time function is not Lipschitz continuous at the origin. This is consistent with Example 2.1 where the settling-time function is not Lipschitz continuous. However, as noted earlier, the settling-time function in Example 2.1 is Hölder continuous at the origin. In contrast, the following example shows that even if the settling-time function is continuous, it may not be Hölder continuous at the origin.

Example 2.3. Consider the system (2.1) with $\mathcal{D}=\{x \in \mathbb{R}:|x|<1\}$ and $f: \mathcal{D} \rightarrow \mathbb{R}$ given by

$$
\begin{aligned}
f(x) & =-x(\ln |x|)^{2}, & & x \in \mathcal{D} \backslash\{0\}, \\
& =0, & & x=0 .
\end{aligned}
$$

The system defined by (2.15) is continuous and has the global semiflow

$$
\begin{aligned}
& \mu(t, x)=\operatorname{sign}(x) e^{\frac{\ln |x|}{1+t \ln |x|}}, \quad t<-\frac{1}{\ln |x|}, \quad x \in \mathcal{D} \backslash\{0\}, \\
& =0, \quad t \geq-\frac{1 \mid}{\ln |x|}, \quad x \in \mathcal{D} \backslash\{0\}, \\
& =0, \quad t \geq 0, \quad x=0 \text {. }
\end{aligned}
$$

From the solution (2.16), it is clear that 0 is a finite-time-stable equilibrium in the neighborhood $\mathcal{N}=\mathcal{D}$ and the settling-time function, which is continuous, is given by

$$
\begin{aligned}
T(x) & =-\frac{1}{\ln |x|}, & & x \in \mathcal{D} \backslash\{0\}, \\
& =0, & & x=0 .
\end{aligned}
$$

Since $\lim _{h \rightarrow 0^{+}} h^{\gamma}|\ln h|=0$ for every $\gamma>0$, it follows that for every $\gamma>0, \frac{T(\cdot)}{|\cdot| \gamma}$ is unbounded in every deleted neighborhood of 0 . Thus $T$ is not Hölder continuous at the origin.

3. Finite-time repellers. The results of this section do not depend upon the assumption of forward uniqueness.

If the origin is not Lyapunov stable, then there exists an open neighborhood $\mathcal{U}$ of the origin and solutions that start arbitrarily close to the origin and eventually leave $\mathcal{U}$. However, in the case of Lipschitzian dynamics, solutions are continuous in the initial condition over bounded time intervals so that solutions with initial conditions sufficiently close to the origin stay in $\mathcal{U}$ for arbitrarily large amounts of time. In the non-Lipschitzian case, where solutions need not be continuous in the initial condition even over a bounded time interval, it is natural to expect the existence of solutions that start arbitrarily close to the origin and yet leave a certain neighborhood in a fixed amount of time. We therefore have the following definition. 
DeFINITION 3.1. The origin is said to be a finite-time repeller if there exists a neighborhood $\mathcal{U} \subset \mathcal{D}$ of the origin and $\tau>0$ such that, for every open neighborhood $\mathcal{V} \subseteq \mathcal{U}$ of the origin, there exists $h \in(0, \tau]$ and a solution $y:[0, h] \rightarrow \mathcal{D}$ of $(2.1)$ such that $y(0) \in \mathcal{V}$ and $y(h) \notin \mathcal{U}$. The origin is said to be a finite-time saddle if the origin is a finite-time repeller in forward as well as reverse time.

Definition 3.1 implies that solutions of (2.1) with initial conditions sufficiently close to a finite-time repeller do not depend continuously on the initial conditions over the bounded time interval $[0, \tau]$. In other words, a system is extremely sensitive to perturbations close to a finite-time repeller. As noted in section 2 , under the assumption of uniqueness, solutions are continuous functions of the initial conditions and hence nonuniqueness is necessary for the existence of a finite-time repeller. The following proposition gives the precise connection between nonuniqueness and finitetime repellers.

Proposition 3.2. The origin is a finite-time repeller if and only if there exist more than one solution of (2.1) originating at the origin.

Proof. Note that $z \equiv 0$ is a solution of (2.1) satisfying $z(0)=0$. To prove sufficiency, suppose $y:[0, \tau] \rightarrow \mathcal{D}, \tau>0$, is a solution of (2.1) such that $y(0)=0$ and $y(\tau) \neq 0$. Then there exists an open set $\mathcal{U} \subset \mathcal{D}$ such that $0 \in \mathcal{U}$ and $y(\tau) \notin \mathcal{U}$. If $\mathcal{V} \subseteq \mathcal{U}$ is an open neighborhood of the origin, then $0=y(0) \in \mathcal{V}$ and $y(h) \notin \mathcal{V}$ for $h=\tau$. Thus the origin is a finite-time repeller.

To prove necessity, suppose that the origin is a finite-time repeller and let $\mathcal{U}$ and $\tau$ be as in Definition 3.1. There exists a sequence $\left\{h_{m}\right\}$ of real numbers in $(0, \tau]$ and a sequence of solutions $y_{m}:\left[0, h_{m}\right] \rightarrow \mathcal{D}$ of $(2.1)$ such that, $y_{m}(0) \rightarrow 0$ as $m \rightarrow \infty$ and $y_{m}\left(h_{m}\right) \notin \mathcal{U}$. Now suppose that $z \equiv 0$ is the unique solution of (2.1) satisfying $z(0)=0$. Then there exists $N>0$ such that for every $m>N, y_{m}$ can be extended to a solution $\hat{y}_{m}$ of $(2.1)$ defined on $[0, \tau]$ and $\hat{y}_{m} \rightarrow z$ uniformly on $[0, \tau]$ [12, Lem. I.3.1]. However, this contradicts the fact that, for every $m, h_{m} \in[0, \tau]$ and $\hat{y}_{m}\left(h_{m}\right)=y_{m}\left(h_{m}\right) \notin \mathcal{U}$. Hence we conclude that $z \equiv 0$ is not the unique solution of (2.1) satisfying $z(0)=0$.

Finite-time repellers are called terminal repellers in [7], [23] and some of the references therein. Reference [5] gives an example of a one-degree-of-freedom Lagrangian system having a finite-time saddle, while in [4] finite-time saddles arise in the controlled double integrator. Proposition 3.2 implies that a system exhibits spontaneous and unpredictable departure from an equilibrium state that is a finite-time repeller. This property of finite-time repellers was used in [5] as an example of indeterminacy in classical dynamics, while [23] and some of the references contained therein postulate finite-time repellers as models of irreversibility and unpredictability in complex systems. Finally, [7] proposed a fast global optimization algorithm which utilizes the tendency of solutions to rapidly escape from a neighborhood of a finite-time repeller.

Sections 3.25 and 3.26 in [1] contain sufficient conditions for (2.1) to possess multiple solutions with the initial value 0 . In view of Proposition 3.2, these conditions can also be used to deduce whether the origin is a finite-time repeller. Therefore, sufficient Lyapunov conditions for the origin to be a finite-time repeller will not be considered in this paper.

4. Lyapunov theory. The upper right Dini derivative of a function $g:[a, b) \rightarrow$ $\mathbb{R}, b>a$, is the function $D^{+} g:[a, b) \rightarrow \overline{\mathbb{R}}$ given by

$$
\left(D^{+} g\right)(t)=\limsup _{h \rightarrow 0^{+}} \frac{1}{h}[g(t+h)-g(t)], t \in[a, b) .
$$


The function $g$ is nonincreasing on $[a, b)$ if and only if $\left(D^{+} g\right)(t) \leq 0$ for all $t \in[a, b)$ $\left[13\right.$, p. 84], [16, p. 347]. If $g$ is differentiable at $t$, then $\left(D^{+} g\right)(t)$ is the ordinary derivative of $g$ at $t$.

If the scalar differential equation $\dot{y}(t)=w(y(t))$ has the global semiflow $\mu$ : $\mathbb{R}_{+} \times \mathbb{R} \rightarrow \mathbb{R}$, where $w: \mathbb{R} \rightarrow \mathbb{R}$ is continuous, and $g:[a, b) \rightarrow \mathbb{R}$ is a continuous function such that $\left(D^{+} g\right)(t) \leq w(g(t))$ for all $t \in[a, b)$, then $g(t) \leq \mu(t, g(a))$ for all $t \in[a, b)$. Proofs and more general versions of this result, which is known as the comparison lemma, can be found in [13, sect. 5.2], [15, sect. 2.5], [16, Chap. IX], and [22, sect. 4]. The comparison lemma will be used along with the scalar system of Example 2.1 in the proofs of the main results of this section and the next.

The following lemma will prove useful in the rest of the development.

Lemma 4.1. Let $V: \mathcal{A} \rightarrow \mathbb{R}$ be a continuous function defined on the open set $\mathcal{A} \subseteq \mathbb{R}^{n}$. Let $\mathcal{B}$ be an open set such that $\overline{\mathcal{B}} \subset \mathcal{A}$, let $\Omega_{\kappa}=\{x \in \mathcal{B}: V(x)<\kappa\}$, where $\kappa<\inf _{z \in \operatorname{bd} \mathcal{B}} V(z)$, and let $p: \mathbb{R} \rightarrow \mathbb{R}$ be a continuous function satisfying $p(\kappa)>0$. If $y:[a, b) \rightarrow \mathcal{A}$ is a continuous function that satisfies $y(a) \in \bar{\Omega}_{\kappa}$ and satisfies

$$
\left(D^{+}(V \circ y)\right)(t) \leq-p(V \circ y(t))
$$

for every $t \in[a, b)$ such that $y(t) \in \mathcal{B}$, then $y(t) \in \Omega_{\kappa}$ for all $t \in(a, b)$.

Proof. The assertion is vacuously true if $\Omega_{\kappa}$ is empty. Therefore, let $y:[a, b) \rightarrow \mathcal{A}$ be a continuous function satisfying the hypotheses in the statement of the lemma. Note that by the choice of $\kappa$ and the continuity of $V$, bd $\Omega_{\kappa} \subseteq\{x \in \mathcal{B}: V(x)=\kappa\}$.

First suppose that $y(a) \in$ bd $\Omega_{\kappa}$. Since $p, V$, and $y$ are continuous and $p(V(y(a)))$ $=p(\kappa)>0$, it follows that there exists $s>0$ such that $p(V(y(t)))>0$ for all $t \in[a, a+s)$. Moreover, $s$ may be chosen such that $y(t) \in \mathcal{B}$ for all $t \in[a, a+s)$. Equation (4.2) now implies that $V \circ y$ is strictly decreasing on $[a, a+s)$ so that $y(t) \in \Omega_{\kappa}$ for all $t \in(a, a+s)$.

Now suppose $y(h) \in \Omega_{\kappa}$ for some $h \in[a, b)$. If $y(t) \notin \Omega_{\kappa}$ for some $t \in[h, b)$, then, by continuity, there exists $\tau \in(h, b)$ such that $y(\tau) \in$ bd $\Omega_{\kappa}$ and $y(t) \in \Omega_{\kappa}$ for all $t \in[h, \tau)$. Therefore, $y$ satisfies $(4.2)$ on $[h, \tau)$. Since $p, V$, and $y$ are continuous and $p(V(y(\tau)))=p(\kappa)>0$, it follows that there exists $s>0$ such that $p(V(y(t)))>0$ for all $t \in[\tau-s, \tau)$. Equation (4.2) now implies that $V \circ y$ is nonincreasing on $[\tau-s, \tau)$ so that $\kappa=V(y(\tau)) \leq V(y(\tau-s))<\kappa$, which is a contradiction. Hence we conclude that $y(t) \in \Omega_{\kappa}$ for all $t \in[h, b)$.

It follows from the above two facts that if $y(a) \in \bar{\Omega}_{\kappa}$, then $y(t) \in \Omega_{\kappa}$ for all $t \in(a, b)$.

Given a continuous function $V: \mathcal{D} \rightarrow \mathbb{R}$, the upper-right Dini derivative of $V$ along the solutions of (2.1) is a $\overline{\mathbb{R}}$-valued function $\dot{V}$ given by

$$
\dot{V}(x)=\left(D^{+}\left(V \circ \psi^{x}\right)\right)(0) .
$$

$\dot{V}(x)$ is defined for every $x \in \mathcal{D}$ for which $\psi^{x}$ is defined. It is easy to see that $\dot{V}(0)$, if defined, is 0 . Also, since $\psi$ is a local semiflow, it can be shown that if $\psi^{x}(t)$ is defined, then

$$
\dot{V}\left(\psi^{x}(t)\right)=\left(D^{+}\left(V \circ \psi^{x}\right)\right)(t) .
$$

It can also be shown that if $V$ is locally Lipschitz at $x \in \mathcal{D} \backslash\{0\}$, then [13, sect. 5.1], $[16$, p. 353], [22, p. 3]

$$
\dot{V}(x)=\limsup _{h \rightarrow 0^{+}} \frac{1}{h}[V(x+h f(x))-V(x)] .
$$


If $V$ is continuously differentiable on $\mathcal{D} \backslash\{0\}$, then (4.3) and (4.5) both yield the Lie derivative

$$
\dot{V}(x)=\frac{d\left(V \circ \psi^{x}\right)}{d t}(0)=\frac{\partial V}{\partial x}(x) f(x), x \in \mathcal{D} \backslash\{0\} .
$$

A function $V: \mathcal{D} \rightarrow \mathbb{R}$ is said to be proper if $V^{-1}(K)$ is compact for every compact set $K \subset \mathbb{R}$. Note that if $\mathcal{D}=\mathbb{R}^{n}$ and $V$ is radially unbounded, then $V$ is proper.

We are now ready to state the main result of this paper. Versions of this result have either appeared without proof or have been used implicitly in [3], [4], [11], [18], [19].

THEOREM 4.2. Suppose there exists a continuous function $V: \mathcal{D} \rightarrow \mathbb{R}$ such that the following conditions hold:

(i) $V$ is positive definite.

(ii) There exist real numbers $c>0$ and $\alpha \in(0,1)$ and an open neighborhood $\mathcal{V} \subseteq \mathcal{D}$ of the origin such that

$$
\dot{V}(x)+c(V(x))^{\alpha} \leq 0, x \in \mathcal{V} \backslash\{0\} .
$$

Then the origin is a finite-time-stable equilibrium of (2.1). Moreover, if $\mathcal{N}$ is as in Definition 2.2 and $T$ is the settling-time function, then

$$
T(x) \leq \frac{1}{c(1-\alpha)} V(x)^{1-\alpha}, x \in \mathcal{N},
$$

and $T$ is continuous on $\mathcal{N}$. If in addition $\mathcal{D}=\mathbb{R}^{n}, V$ is proper, and $\dot{V}$ takes negative values on $\mathbb{R}^{n} \backslash\{0\}$, then the origin is a globally finite-time-stable equilibrium of (2.1).

Proof. Since $V$ is positive definite and $\dot{V}$ takes negative values on $\mathcal{V} \backslash\{0\}$, it follows that $y \equiv 0$ is the unique solution of $(2.1)$ on $\mathbb{R}_{+}$satisfying $y(0)=0$ [1, sect. 3.15] [22, Thm. 1.2, p. 5]. Thus every initial condition in $\mathcal{D}$ has a unique solution in forward time. Moreover, $\dot{V}(0)=0$ and thus (4.7) holds on $\mathcal{V}$.

Let $\mathcal{U} \subseteq \mathcal{V}$ be a bounded open set such that $0 \in \mathcal{U}$ and $\overline{\mathcal{U}} \subset \mathcal{D}$. Then bd $\mathcal{U}$ is compact and $0 \notin \mathrm{bd} \mathcal{U}$. The continuous function $V$ attains a minimum on bd $\mathcal{U}$ and by positive definiteness, $\min _{x \in \mathrm{bd}} \mathcal{U} V(x)>0$. Let $0<\beta<\min _{x \in \mathrm{bd}} \mathcal{U} V(x)$ and $\mathcal{N}=\{x \in \mathcal{U}: V(x)<\beta\} . \mathcal{N}$ is nonempty since $0 \in \mathcal{N}$, open since $V$ is continuous, and bounded since $\mathcal{U}$ is bounded.

Now, consider $x \in \mathcal{N}$ and let $c$ and $\alpha$ be as in the theorem statement above. By uniqueness, $\psi^{x}:\left[0, \tau_{x}\right) \rightarrow \mathcal{D}$ is the unique right maximally defined solution of $(2.1)$ for the initial condition $x$. For every $t \in\left[0, \tau_{x}\right)$ such that $\psi^{x}(t) \in \mathcal{U},(4.4)$ and (4.7) yield

$$
\left(D^{+}\left(V \circ \psi^{x}\right)\right)(t) \leq-c\left(V \circ \psi^{x}(t)\right)^{\alpha} .
$$

Thus $y=\psi^{x}$ satisfies the hypotheses of Lemma 4.1 with $\mathcal{A}=\mathcal{D}, \mathcal{B}=\mathcal{U}, \kappa=\beta$, $\Omega_{\kappa}=\mathcal{N}$, and $p(h)=c h^{\alpha}$ for $h \in \mathbb{R}_{+}$. Therefore, by Lemma $4.1, \psi^{x}(t) \in \mathcal{N}$ for all $t \in\left[0, \tau_{x}\right)$. Now $\psi^{x}$ satisfies the hypotheses of Proposition 2.1 with $\mathcal{K}=\mathcal{N}$. Therefore, by Proposition 2.1, $\psi^{x}$ is defined and satisfies (4.9) on $\mathbb{R}_{+}$. Thus $\psi: \mathbb{R}_{+} \times \mathcal{N} \rightarrow \mathcal{N}$ is a continuous global semiflow satisfying (2.3) and (2.4).

Next, applying the comparison lemma to the differential inequality (4.9) and the scalar differential equation (2.7) yields

$$
V(\psi(t, x)) \leq \mu(t, V(x)), t \in \mathbb{R}_{+}, x \in \mathcal{N},
$$


where $\mu$ is given by (2.8) with $k=c$. From (2.8), (4.10), and the positive-definiteness of $V$, we conclude that

$$
\psi(t, x)=0, t \geq \frac{1}{c(1-\alpha)}(V(x))^{1-\alpha}, x \in \mathcal{N} .
$$

Since $\psi(0, x)=x$ and $\psi$ is continuous, $\inf \left\{t \in \mathbb{R}_{+}: \psi(t, x)=0\right\}>0$ for $x \in$ $\mathcal{N} \backslash\{0\}$. Also, it follows from (4.11) that $\inf \left\{t \in \mathbb{R}_{+}: \psi(t, x)=0\right\}<\infty$ for $x \in \mathcal{N}$. Define $T: \mathcal{N} \rightarrow \mathbb{R}_{+}$by using (2.6). It is a simple matter to verify that $T$ and $\mathcal{N}$ satisfy (i) of Definition 2.2 and thus $T$ is the settling-time function on $\mathcal{N}$. Lyapunov stability follows by noting from (4.7) that $\dot{V}$ takes negative values on $\mathcal{V} \backslash\{0\}$. Equation (4.8) follows from (4.11) and (2.6). Equation (4.8) implies that $T$ is continuous at the origin and hence, by Proposition 2.4, continuous on $\mathcal{N}$.

If $\mathcal{D}=\mathbb{R}^{n}$ and $V$ is proper, then global finite-time-stability is proven in the same way that global asymptotic stability is proven using radially unbounded Lyapunov functions. See, for instance, [15, Thm. 3.2], [22, Thm. 11.5].

Remark 4.1. It is difficult to compute $\dot{V}$ by using (4.3) unless solutions to (2.1) are known. Thus, in practice, it will often be more convenient to apply Theorem 4.2 with a Lipschitz continuous or a continuously differentiable function $V$ so that $\dot{V}$ is given by (4.5) or (4.6), respectively.

Theorem 4.2 implies that for a system with a finite-time-stable equilibrium and a discontinuous settling-time function, such as the system considered in Example 2.2, there does not exist a Lyapunov function satisfying the hypotheses of Theorem 4.2. In the case that the settling-time function is continuous, the following theorem provides a converse to the previous one.

THEOREM 4.3. Suppose the origin is a finite-time-stable equilibrium of (2.1) and the settling-time function $T$ is continuous at 0. Let $\mathcal{N}$ be as in Definition 2.2 and let $\alpha \in(0,1)$. Then there exists a continuous function $V: \mathcal{N} \rightarrow \mathbb{R}$ such that the following conditions are satisfied:

(i) $V$ is positive definite.

(ii) $\dot{V}$ is real valued and continuous on $\mathcal{N}$ and there exists $c>0$ such that

$$
\dot{V}(x)+c(V(x))^{\alpha} \leq 0, x \in \mathcal{N} .
$$

Proof. By Proposition 2.4, the settling-time function $T: \mathcal{N} \rightarrow \mathbb{R}_{+}$is continuous. Define $V: \mathcal{N} \rightarrow \mathbb{R}_{+}$by $V(x)=(T(x))^{\frac{1}{1-\alpha}}$. Then $V$ is continuous and positive definite and, by $(2.5), \dot{V}(0)=0$. For $x \in \mathcal{N} \backslash\{0\},(2.10)$ implies that $V \circ \psi^{x}$ is continuously differentiable on $[0, T(x))$ so that (4.3) can be easily computed as $\dot{V}(x)=$ $-\frac{1}{1-\alpha}(T(x))^{\frac{\alpha}{1-\alpha}}=-\frac{1}{1-\alpha}(V(x))^{\alpha}$. Thus $\dot{V}$ is real valued, continuous, and negative definite on $\mathcal{N}$ and satisfies $\dot{V}(x)+c(V(x))^{\alpha}=0$ for all $x \in \mathcal{N}$ with $c=\frac{1}{1-\alpha}$.

Equation (4.8) implies that if $V$ in Theorem 4.2 is Hölder continuous at 0 then so is $T$. However, as shown by Example 2.3, the settling-time function need not be Hölder continuous at the origin. Thus the conclusion regarding the continuity of $V$ in Theorem 4.3 cannot be strengthened to Hölder continuity. In particular, the scalar system considered in Example 2.3, where $T$ is not Hölder continuous, does not admit a continuously differentiable or Lipschitz continuous Lyapunov function that satisfies the hypotheses of Theorem 4.2, since either Lipschitz continuity or differentiability implies Hölder continuity. As the next section shows, the existence of Lipschitz continuous Lyapunov functions is of importance in studying the behavior of finite-time-stable systems in the presence of perturbations. 
5. Sensitivity to perturbations. In a realistic problem, (2.1) might represent a nominal model that is valid only under ideal conditions, while a more accurate description of the system might be provided by the perturbed model

$$
\dot{y}(t)=f(y(t))+g(t, y(t)),
$$

where the perturbation term $g$ results from disturbances, uncertainties, parameter variations, or modelling errors. In this section we investigate the sensitivity to perturbations of systems with a finite-time-stable equilibrium by studying the behavior of solutions of the perturbed system (5.1) in a neighborhood of the finite-time-stable equilibrium of the nominal system (2.1).

For simplicity, we consider only continuous perturbation terms $g: \mathbb{R}_{+} \times \mathcal{D} \rightarrow \mathbb{R}^{n}$ so that the local existence of solutions of the perturbed system (5.1) is guaranteed. Right maximally defined solutions of (5.1) are defined as in section 2. We will need the following extension of Proposition 2.1 to time-varying systems. Proofs appear in [12, pp. 17-18], [22, Thm. 3.3, p. 12].

Proposition 5.1. If $y:[0, \tau) \rightarrow \mathcal{D}$ is a right maximally defined solution of (5.1) such that $y(t) \in \mathcal{K}$ for all $t \in[0, \tau)$, where $\overline{\mathcal{K}} \subset \mathcal{D}$ is compact, then $\tau=\infty$.

If $y:[0, \tau) \rightarrow \mathcal{D}$ is a solution of (5.1) and $V: \mathcal{D} \rightarrow \mathbb{R}$ is Lipschitz continuous on $\mathcal{D}$ with Lipschitz constant $M$, then it can be shown that

$$
\left(D^{+}(V \circ y)\right)(t) \leq \dot{V}(y(t))+M\|g(t, y(t))\|, t \in[0, \tau),
$$

where $\dot{V}$ is computed along the solutions of the unperturbed system (2.1) using equation (4.3). See, for instance, the proof of Lemma X.5.1 in [12].

The following theorem concerns the behavior of finite-time-stable systems under bounded perturbations. Such perturbations, include, as a special case, bounded persistent disturbances of the form $g(t, y(t))=v(t)$.

TheOREm 5.2. Suppose there exists a function $V: \mathcal{D} \rightarrow \mathbb{R}$ such that $V$ is positive definite and Lipschitz continuous on $\mathcal{D}$, and satisfies (4.7), where $\mathcal{V} \subseteq \mathcal{D}$ is an open neighborhood of the origin, $c>0$ and $\alpha \in\left(0, \frac{1}{2}\right)$. Then there exist $\delta_{0}>0, l>0$, $\Gamma>0$, and an open neighborhood $\mathcal{U}$ of the origin such that, for every continuous function $g: \mathbb{R}_{+} \times \mathcal{D} \rightarrow \mathbb{R}^{n}$ with

$$
\delta=\sup _{\mathbb{R}_{+} \times \mathcal{D}}\|g(t, x)\|<\delta_{0}
$$

every right maximally defined solution $y$ of (5.1) with $y(0) \in \mathcal{U}$ is defined on $\mathbb{R}_{+}$and satisfies $y(t) \in \mathcal{U}$ for all $t \in \mathbb{R}_{+}$and

$$
\|y(t)\| \leq l \delta^{\gamma}, t \geq \Gamma
$$

where $\gamma=\frac{1-\alpha}{\alpha}>1$.

Proof. By Theorem 4.2, the origin is a finite-time-stable equilibrium for (2.1). Let $\mathcal{N}$ be as in Definition 2.2 and let $T: \mathcal{N} \rightarrow \mathbb{R}^{+}$be the settling-time function. By Proposition 2.4, there exists $r>0$ and an open neighborhood $\mathcal{U}_{r} \subset \mathcal{N} \cap \mathcal{V}$ of 0 such that $T(x) \geq r\|x\|$ for $x \in \mathcal{U}_{r}$. Also, by Theorem 4.2, $T$ satisfies (4.8). Without any loss of generality, we assume that $\overline{\mathcal{U}}_{r}$ is compact and $\overline{\mathcal{U}}_{r} \subset \mathcal{N} \cap \mathcal{V}$. Let $\mathcal{U}=\left\{x \in \mathcal{U}_{r}: V(x)<\beta\right\}$, where $0<\beta<\min _{z \in \text { bd } \mathcal{U}_{r}} V(z)$. Then $\mathcal{U}$ is nonempty, open, and bounded.

Let $M>0$ be the Lipschitz constant of $V$ and let $\delta_{0}>0$ satisfy $c \beta^{\alpha}-2 M \delta_{0}>0$. Suppose $g: \mathbb{R}_{+} \times \mathcal{D} \rightarrow \mathbb{R}^{n}$ is a continuous function that satisfies (5.3) and consider a 
right maximally defined solution $y:[0, \tau) \rightarrow \mathcal{D}$ of $(5.1)$ with $x=y(0) \in \mathcal{U}$. Equations (4.7), (5.2), and (5.3) imply that for every $t \in[0, \tau)$ such that $y(t) \in \mathcal{U}_{r}$,

$$
\left(D^{+}(V \circ y)\right)(t) \leq-c(V(y(t)))^{\alpha}+M \delta .
$$

Since $c \beta^{\alpha}-M \delta>c \beta^{\alpha}-2 M \delta>c \beta^{\alpha}-2 M \delta_{0}>0$, (5.5) implies that $y$ satisfies the hypotheses of Lemma 4.1 with $\mathcal{A}=\mathcal{D}, \mathcal{B}=\mathcal{U}_{r}, \kappa=\beta, \Omega_{\kappa}=\mathcal{U}$, and $p(h)=c h^{\alpha}-M \delta$ for $h \in \mathbb{R}_{+}$. Therefore, Lemma 4.1 implies that $y(t) \in \mathcal{U}$ for $t \in[0, \tau)$. The right maximally defined solution $y$ satisfies the hypotheses of Proposition 5.1 with $\mathcal{K}=\mathcal{U}$. Thus, by Proposition 5.1, $y$ is defined on $\mathbb{R}_{+}$and (5.5) holds on $\mathbb{R}_{+}$.

Now, let $\mathcal{W}=\left\{x \in \mathcal{U}: V(x)<\left(\frac{2 M \delta}{c}\right)^{\frac{1}{\alpha}}\right\}$. If $y(\tau) \in \overline{\mathcal{W}}$ for some $\tau>0$, then (5.5) implies that $y$ satisfies the hypotheses of Lemma 4.1 on $[\tau, \infty)$ with $\mathcal{A}=\mathcal{U}_{r}, \mathcal{B}=\mathcal{U}$, $\kappa=\left(\frac{2 M \delta}{c}\right)^{\frac{1}{\alpha}}, \Omega_{\kappa}=\mathcal{W}$, and $p(h)=c h^{\alpha}-M \delta$ for $h \in \mathbb{R}_{+}$, and hence $y(t) \in \mathcal{W}$ for all $t>\tau$. Therefore, suppose $y(0)=x \notin \overline{\mathcal{W}}$ so that $y^{-1}(\mathcal{W})$, which is open by continuity, is of the form $\left(t_{x}, \infty\right)$ with $t_{x}>0$. Since $y(t) \notin \mathcal{W}$ for all $t \in\left[0, t_{x}\right]$, it follows that $V(y(t)) \geq\left(\frac{2 M \delta}{c}\right)^{\frac{1}{\alpha}}$ for all $t \in\left[0, t_{x}\right]$. Equation (5.5) now implies that

$$
\left(D^{+}(V \circ y)\right)(t) \leq-\frac{1}{2} c(V(y(t)))^{\alpha}, t \in\left[0, t_{x}\right) .
$$

Applying the comparison principle to the differential inequality (5.6) and the scalar differential equation (2.7) we obtain

$$
(V \circ y)(t) \leq \mu(t, V(x)), t \in\left[0, t_{x}\right),
$$

where $\mu$ is given by (2.8) with $k=\frac{1}{2} c$. By continuity, the inequality (5.7) also holds for $t=t_{x}$. Since $V\left(y\left(t_{x}\right)\right) \geq\left(\frac{2 M \delta}{c}\right)^{\frac{1}{\alpha}}>0$, the comparison (5.7) yields $\mu\left(t_{x}, V(x)\right)>$ 0 . Equation (2.8) now gives $t_{x}<\frac{2}{c(1-\alpha)}(V(x))^{1-\alpha}<\frac{2}{c(1-\alpha)} \beta^{1-\alpha}$. Thus $V(y(t))$ $<\left(\frac{2 M \delta}{c}\right)^{\frac{1}{\alpha}}$ for $t \geq \Gamma \triangleq \frac{2}{c(1-\alpha)} \beta^{1-\alpha}$. It now follows from (2.11) and (4.8) that for $t>\Gamma$,

$$
\|y(t)\| \leq \frac{1}{r c(1-\alpha)}(V(y(t)))^{1-\alpha} \leq \frac{1}{r c(1-\alpha)}\left(\frac{2 M \delta}{c}\right)^{\frac{1-\alpha}{\alpha}}
$$

Equation (5.4) now follows by choosing $l \triangleq \frac{1}{r c(1-\alpha)}\left(\frac{2 M}{c}\right)^{\frac{1-\alpha}{\alpha}}>0$.

Note that in Theorem 4.3, $\alpha$ can be chosen to be arbitrarily small. Hence the requirement in Theorem 5.2 that $\alpha$ lie in $\left(0, \frac{1}{2}\right)$ is not restrictive. This choice of $\alpha$ leads to $\gamma>1$ in (5.4) which implies that for $\delta$ in equation (5.3) sufficiently small, the ultimate bound (5.4) on the state is of higher order than the bound on the perturbation. In analogous theorems on exponential stability for Lipschitzian systems, $\alpha$ in equation (4.7) is at least 1 [15, Thm. 3.12], [22, Thm. 19.2] while $\gamma$ in (5.4) is at most 1 [15, Lemma 5.2]. Thus for a Lipschitzian system with an exponentially stable equilibrium at the origin, the ultimate bound on the state can only be guaranteed to be of the same order of magnitude as the perturbation and not less. Consequently, finite-time stability of the origin leads to improved rejection of low-level persistent disturbances.

The following theorem deals with perturbations that are globally Lipschitz in the state variables uniformly in time. Such perturbations might represent model uncertainties. 
TheOREm 5.3. Suppose there exists a function $V: \mathcal{D} \rightarrow \mathbb{R}$ such that $V$ is positive definite and Lipschitz continuous on $\mathcal{D}$, and satisfies (4.7), where $\mathcal{V} \subseteq \mathcal{D}$ is an open neighborhood of the origin, $c>0$ and $\alpha \in\left(0, \frac{1}{2}\right)$. Then, for every $L \geq 0$, there exists an open neighborhood $\mathcal{U}$ of the origin and $\Gamma>0$ such that, for every continuous function $g: \mathbb{R}_{+} \times \mathcal{D} \rightarrow \mathbb{R}^{n}$ satisfying

$$
\|g(t, x)\| \leq L\|x\|, \quad(t, x) \in \mathbb{R}_{+} \times \mathcal{D},
$$

every right maximally defined solution $y$ of (5.1) with $y(0) \in \mathcal{U}$ is defined on $\mathbb{R}_{+}$and satisfies $y(t) \in \mathcal{U}$, for all $t \in \mathbb{R}_{+}$, and $y(t)=0$ for all $t \geq \Gamma$.

Proof. By Theorem 4.2, the origin is a finite-time-stable equilibrium for (2.1). Let $\mathcal{N}$ be as in Definition 2.2 and let $T: \mathcal{N} \rightarrow \mathbb{R}_{+}$be the settling-time function. Fix $L \geq 0$ and let $r>0$ be such that $c[r(1-\alpha)]^{\alpha}>(2 M L)^{1-\alpha}$, where $M>0$ is the Lipschitz constant of $V$. By Proposition 2.4, there exists an open set $\mathcal{U}_{r} \subset \mathcal{N} \cap \mathcal{V}$ such that $r\|x\| \leq T(x)$ for all $x \in \mathcal{U}_{r}$. Also, by Theorem 4.2, $T$ satisfies (4.8). Without any loss of generality, we may assume that $\|x\|<1$ for $x \in \mathcal{U}_{r}$ and $\overline{\mathcal{U}}_{r} \subset \mathcal{N} \cap \mathcal{V}$. Let $\mathcal{U}=\left\{x \in \mathcal{U}_{r}: V(x)<\beta\right\}$, where $0<\beta<\min _{z \in \operatorname{bd}} \mathcal{U}_{r} V(z)$. Note that $\mathcal{U}$ is nonempty, open, and bounded. Also, $\frac{\alpha}{1-\alpha}<1$ so that $\|x\| \leq\|x\|^{\frac{\alpha}{1-\alpha}}$ for $\|x\|<1$. Therefore, (2.11) and (4.8) yield

$$
2 M L\|x\| \leq c[r c(1-\alpha)\|x\|]^{\frac{\alpha}{1-\alpha}} \leq c[c(1-\alpha) T(x)]^{\frac{\alpha}{1-\alpha}} \leq c(V(x))^{\alpha}, x \in \mathcal{U}_{r}
$$

Next, let $x \in \mathcal{U}$ and let $g: \mathbb{R}_{+} \times \mathcal{D} \rightarrow \mathbb{R}^{n}$ be a continuous function satisfying (5.8). Consider a right maximally defined solution $y:[0, \tau) \rightarrow \mathcal{D}$ of (5.1) such that $y(0)=x$. For every $t \in[0, \tau)$ such that $y(t) \in \mathcal{U}_{r},(4.7),(5.2)$, and (5.8) yield

$$
\left(D^{+}(V \circ y)\right)(t) \leq-c(V \circ y(t))^{\alpha}+M L\|y(t)\| .
$$

Using (5.9) in (5.10) we obtain

$$
\left(D^{+}(V \circ y)\right)(t) \leq-\frac{c}{2}(V \circ y(t))^{\alpha}, y(t) \in \mathcal{U}_{r} .
$$

Lemma 4.1 now applies with $\mathcal{A}=\mathcal{D}, \mathcal{B}=\mathcal{U}_{r}, \kappa=\beta, \Omega_{\kappa}=\mathcal{U}$, and $p(h)=\frac{c}{2} h^{\alpha}$ for $h \in \mathbb{R}_{+}$so that $y(t) \in \mathcal{U}$ for $t \in[0, \tau)$. The hypotheses of Proposition 5.1 are now satisfied by the right maximally defined solution $y$ of (5.1) with $\mathcal{K}=\mathcal{U}$. Hence, by Proposition 5.1, $\tau=\infty$ and (5.11) holds on $\mathbb{R}_{+}$. Applying the comparison principle to the differential inequality (5.11) and the scalar differential equation (2.7) yields

$$
(V \circ y)(t) \leq \mu(t, V(x)), t \in \mathbb{R}_{+},
$$

where $\mu$ is given by (2.8) with $k=\frac{1}{2} c$. Equation (2.8) and the inequality (5.12) imply that $y(t)=0$ for $t \geq \Gamma \triangleq \frac{2 \beta^{1-\alpha}}{c(1-\alpha)}$.

The following theorem specializes Theorem 5.3 to time-invariant perturbations and shows that finite-time stability is preserved under Lipschitzian perturbations.

TheOrem 5.4. Suppose there exists a function $V: \mathcal{D} \rightarrow \mathbb{R}$ such that $V$ is positive definite and Lipschitz continuous on $\mathcal{D}$ and satisfies (4.7), where $\mathcal{V} \subseteq \mathcal{D}$ is an open neighborhood of the origin, $c>0$, and $\alpha \in\left(0, \frac{1}{2}\right)$. Let $g: \mathcal{D} \rightarrow \mathbb{R}^{n}$ be Lipschitz 
continuous on $\mathcal{D}$ and such that the differential equation

$$
\dot{y}(t)=f(y(t))+g(y(t))
$$

possesses unique solutions in forward time for initial conditions in $\mathcal{D} \backslash\{0\}$. Then the origin is a finite-time-stable equilibrium of (5.13).

Proof. Using steps similar to those used in deriving (5.11) above, it can be shown that $\dot{V}(x) \leq-\frac{c}{2}(V(x))^{\alpha}$ for all $x$ in some open neighborhood of the origin, where $\dot{V}$ denotes the upper-right Dini derivative of $V$ along the solutions of (5.13). Finite-time stability now follows from Theorem 4.2.

The existence of a Lipschitz continuous function satisfying the hypotheses of Theorem 5.4 is sufficient but not necessary for the conclusions to hold. For instance, consider a scalar system of the form (5.13) where the nominal dynamics $f$ are given by (2.15) in Example 2.3, and $g: \mathcal{D} \rightarrow \mathbb{R}$ is Lipschitz continuous on $\mathcal{D}=\{x \in \mathbb{R}:|x|<1\}$ with Lipschitz constant $L$. As noted at the end of section 4 , the nominal dynamics do not admit a Lipschitz continuous Lyapunov function satisfying the hypotheses of Theorem 5.4. However, the origin is still a finite-time-stable equilibrium for the perturbed system. This can be verified by considering the continuous Lyapunov function $V(x)=(\ln |x|)^{-2}, x \in \mathcal{D} \backslash\{0\}, V(0)=0$. It is easy to compute $\dot{V}$ along the solutions of the perturbed system (5.13) for $x \neq 0$ and establish that $\dot{V}(x)<-\sqrt{V(x)}$ for $0<|x|<e^{-\sqrt{2 L}}$, thus proving finite-time stability by Theorem 4.2. This indicates that the main results of this section may be valid under the weaker assumption of finite-time stability with a continuous settling-time function. From the point of view of stability theory, proofs of these results under such weaker hypotheses are certainly of interest. However, as observed in Remark 4.1, the Lyapunov functions used to verify stability properties are often continuously differentiable in practice. In such a case, the results of this section are immediately applicable.

6. Conclusions. The notion of finite-time stability can be precisely formulated within the framework of continuous autonomous systems with forward uniqueness. These assumptions, however, do not imply any regularity properties for the settlingtime function, which may be discontinuous or continuous yet Hölder discontinuous.

Lyapunov and converse Lyapunov results for finite-time stability naturally involve finite-time scalar differential inequalities. The regularity properties of a Lyapunov function satisfying such an inequality strongly depend on the regularity properties of the settling-time function.

Under the assumption of the existence of a Lipschitz continuous Lyapunov function, finite-time stability leads to better rejection of persistent as well as vanishing perturbations. Such an assumption, however, is not strictly necessary, as the discussion at the end of section 5 shows.

The paper thus raises certain questions that are important from the point of view of stability theory. In particular, conditions on the dynamics for the settling-time function to be Hölder continuous and conditions on the settling-time function that lead to a stronger converse result than Theorem 4.3 are of interest. Also of interest are results similar to those given in section 5 but with weaker hypotheses.

As mentioned earlier, a control system under the action of a time-optimal feedback controller yields a closed-loop system that exhibits finite-time convergence. Hence it would be interesting to explore the connections between finite-time-stability and time optimality and relate the results of this paper to results on the time-optimal control problem. 


\section{REFERENCES}

[1] R. P. Agarwal and V. Lakshmikantham, Uniqueness and Nonuniqueness Criteria for Ordinary Differential Equations, Ser. Real Anal. 6, World Scientific, Singapore, 1993.

[2] M. Athans and P. L. Falb, Optimal Control: An Introduction to the Theory and Its Applications, McGraw-Hill, New York, 1966.

[3] S. P. Bhat and D. S. Bernstein, Lyapunov Analysis of Finite-Time Differential Equations, in Proceedings of the American Control Conference, Seattle, WA, 1995, pp. 1831-1832.

[4] S. P. Bhat And D. S. Bernstein, Continuous, finite-time stabilization of the translational and rotational double integrators, IEEE Trans. Automat. Control, 43 (1998), pp. 678-682.

[5] S. P. Bhat And D. S. Bernstein, Example of indeterminacy in classical dynamics, Internat. J. Theoret. Phys., 36 (1997), pp. 545-550.

[6] N. P. Bhatia and O. Hajek, Local Semi-Dynamical Systems, Lecture Notes in Math. 90, Springer-Verlag, Berlin, 1969.

[7] B. C. Cetin, J. Barhen, And J. W. Burdick, Terminal repeller unconstrained subenergy tunneling (TRUST) for fast global optimization, J. Optim. Theory Appl., 77 (1993), pp. 97126.

[8] J.-M. Coron, On the stabilization in finite time of locally controllable systems by means of continuous time-varying feedback law, SIAM J. Control Optim., 33 (1995), pp. 804-833.

[9] A. F. Filippov, Differential Equations with Discontinuous Righthand Sides, Math. Appl., Kluwer Academic Publishers, Dordrecht, the Netherlands, 1988.

[10] I. FlüGGe-Lotz, Discontinuous and Optimal Control, McGraw-Hill, New York, 1968.

[11] V. T. HAImo, Finite time controllers, SIAM J. Control Optim., 24 (1986), pp. 760-770.

[12] J. K. Hale, Ordinary Differential Equations, 2nd ed., Pure and Applied Mathematics XXI, Krieger, Malabar, FL, 1980.

[13] A. G. Kartsatos, Advanced Ordinary Differential Equations, Mariner, Tampa, FL, 1980.

[14] M. Kawski, Stabilization of nonlinear systems in the plane, Systems Control Lett., 12 (1989), pp. 169-175.

[15] H. K. Khalil, Nonlinear Systems, 2nd ed., Tr. Prentice-Hall, Upper Saddle River, NJ, 1996.

[16] N. Rouche, P. Habets, And M. Laloy, Stability Theory by Liapunov's Direct Method, Appl. Math. Sciences, Springer-Verlag, New York, 1977.

[17] E. P. RYAn, Optimal Relay and Saturating Control System Synthesis, IEE Control Engrg. Ser. 14, Peter Peregrinus Ltd., London, 1982.

[18] E. P. RYAn, Finite-time stabilization of uncertain nonlinear planar systems, Dynam. Control, 1 (1991), pp. 83-94.

[19] S. V. SAlEhi AND E. P. RYAn, On optimal nonlinear feedback regulation of linear plants, IEEE Trans. Automat. Control, 26 (1982), pp. 1260-1264.

[20] G. R. Sell, Topological Dynamics and Ordinary Differential Equations, Van Nostrand Reinhold, London, 1971.

[21] S. T. Venkataraman and S. Gulati, Terminal slider control of nonlinear systems, in Proceedings of the International Conference on Advanced Robotics, Pisa, Italy, 1990.

[22] T. Yoshizawa, Stability Theory by Liapunov's Second Method, the Mathematical Society of Japan, Tokyo, 1966.

[23] M. ZAK, Introduction to terminal dynamics, Complex Systems, 7 (1993), pp. 59-87. 